\title{
A case of the appendix myxomas
}

\section{Osamu Tanaka*}

Gifu Municipal Hospital, Department of Radiation Oncology, Japan

Case: 64 years old, men.

Chief complaint: Stomachache.

Clinical history: Because of chronic renal failure, we are treating it by long-term dialysis. Because we reported stomachache, after performing $\mathrm{CT}$, a mass lesion was detected in the ileocecum.

Laboratory findings: There is no that you should mention specially. Image findings

Simple CT: $3^{\star} 2 \mathrm{~cm}$ major oval space-occupying lesions are detected in the ileocecum. The internal resorption level is relatively uniform and is associated with the calcification of the wall partly (Figure 1).

Cystography CT: We show it, but there are no mild augmentation effects in the internal augmentation effect to wall. We contact with a right external iliac artery, but cannot point out the clear invasion (Figure 2).

MRI: T1WI: With the low signal which is relatively uniform in the inside (Figure 3).

T2WI: With the high signal which is relatively uniform in the inside (Figure 4).

Fat suppression T2WI: The inside presents a high signal and cannot point out the region with the signal decrease as compared with T2WI (Figure 5).

\section{Discussion}

Viscous liquid accumulates in the peripheral appendix by appendix lumen occluding for some kind of causes aseptically, and appendiceal some or all is in condition to have expanded it, and most are monolocular. The thing appealing for stomachache has the thing

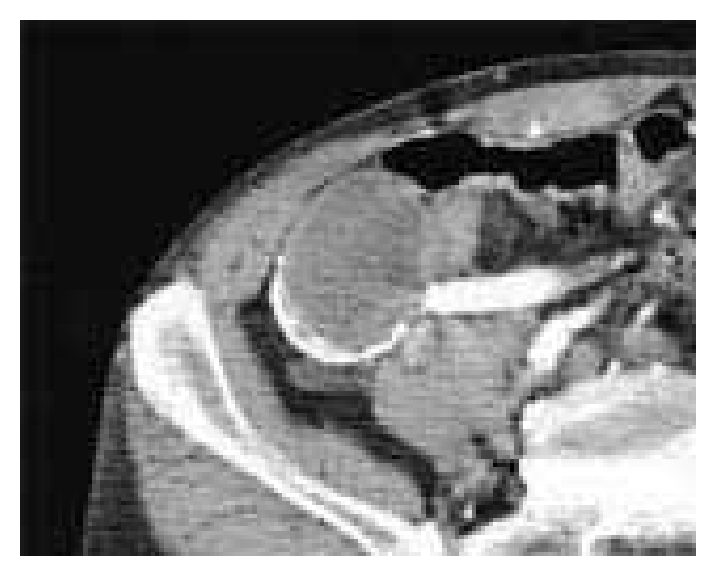

Figure 1. Plain CT discovered accidentally clinically. By the failure of the bulla wall, we may form peritoneal.

Typically, appendix is not contrasted, and a hemispheric smooth eminence projecting into the lumen from the inferior pole of cecum is found in colonic enema examination with contrast material. Also, the orbicular shadow of "the head strap form" is seen in the middle of the eminence when we see an upheaval in detail. We may show the calcification of the wall.

The colonoscopy reveals it as the torose lesion that the wide basis-related surface is smooth in the inferior pole of cecum. The

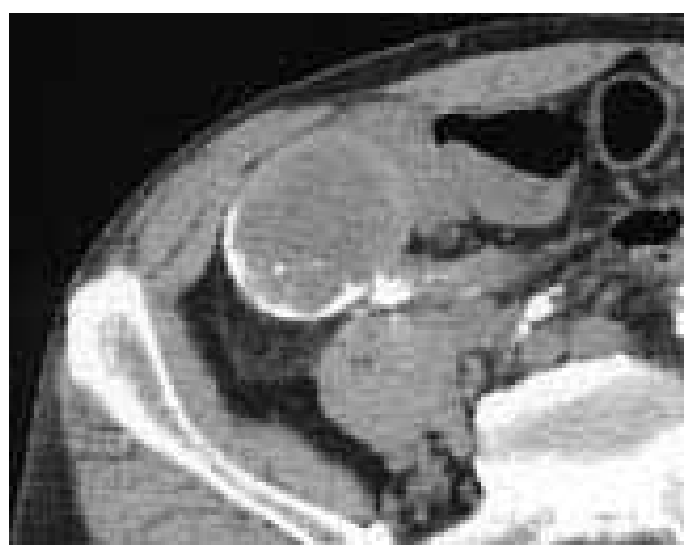

Figure 2. Contrast enhanced CT

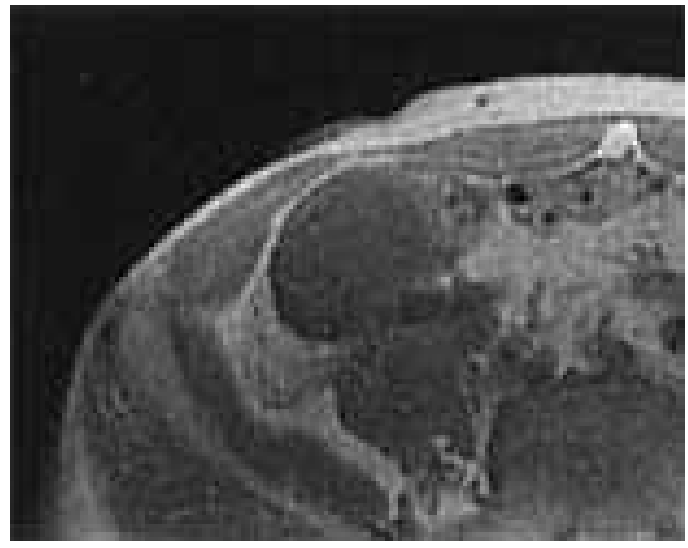

Figure 3. T1-weighted MR image

Correspondence to: Dr. Osamu Tanaka, Gifu Municipal Hospital, Department of Radiation Oncology, japan; E-mail:c.bluered@gmail.com

Received: March 25, 2017; Accepted: April 21, 2017; Published: April 24, 2017 


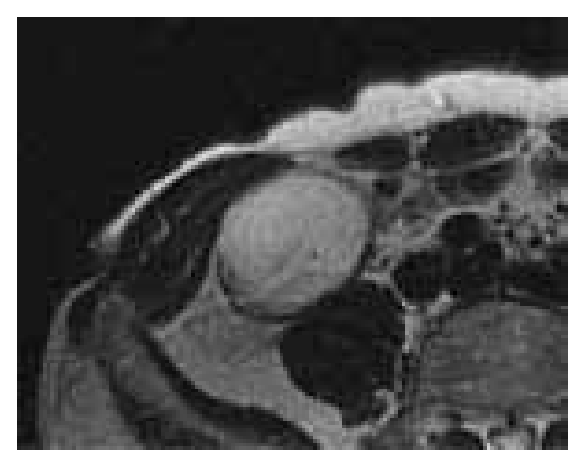

Figure 4. T2-weighted MR image

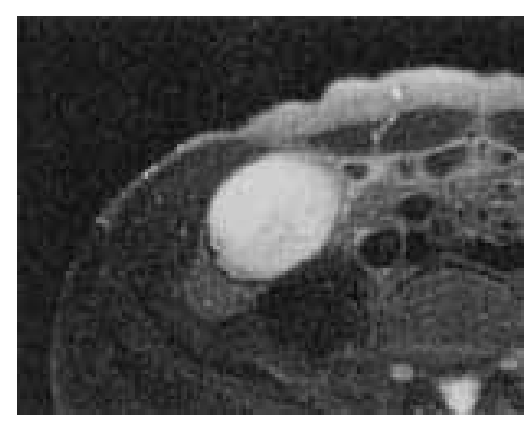

Figure 5. Fat suppressed T1-weighted image differentiation with the submucosal tumors such as lipomas becomes the problem. However, it becomes the clue which the disease is suspected in if we show a head strap-formed all circumferencesrelated pleat.

There were no typical colonic enema findings in this case, but appendix myxomas became suspected in the ileocecum as a bullarelated lesion with the calcification.

\section{References}

1. Pickhardt PJ, Levy AD, Rohrmann CA Jr, Kende AI (2003) Primary neoplasms of the appendix: Radiologic spectrum of disease with pathologic correlation. Radiographics 23: 645-662. [Crossref]

2. Pickhardt PJ, Levy AD, Rohrmann CA Jr, Kende AI (2002) Primary neoplasms of the appendix manifesting as acute appendicitis: CT Findings with pathologic comparison. Radiology 224: 775-781. [Crossref]

3. Karakuc RJ (1967) Unusual roentgenographic manifestations of mucocele of the appendix. Radiology 89: 886-887. [Crossref]

4. Kim SH, Lim HK, Lee WJ, Lim JH, Byun JY (1998) Mucocele of the appendix: ultrasonographic and CT findings. Abdom Imaging 23: 292-296. [Crossref]

5. Shukunami K, Kaneshima NM, Kotsuji F (2000) Preoperative diagnosis and radiographic findings of a freely movable mucocele of the vermiform appendix. Can Assoc Radiol J 51: 281-282. [Crossref]

6. Euphrat EJ (1947) Roentgen features of mucocele of the appendix. Radiology 48: 113117. [Crossref]

Copyright: (C2017 Tanaka O. This is an open-access article distributed under the terms of the Creative Commons Attribution License, which permits unrestricted use, distribution, and reproduction in any medium, provided the original author and source are credited. 